Document downloaded from:

http://hdl.handle.net/10251/65018

This paper must be cited as:

Blandez Barradas, JF.; Esteve Adell, I.; Primo Arnau, AM.; Alvaro Rodríguez, MM.; García Gómez, H. (2015). Palladium nanoparticles supported on graphene as catalysts for the dehydrogenative coupling of hydrosilanes and amines. Catalysis Science and Technology. 5(4):2167-2173. doi:10.1039/c4cy01486c.

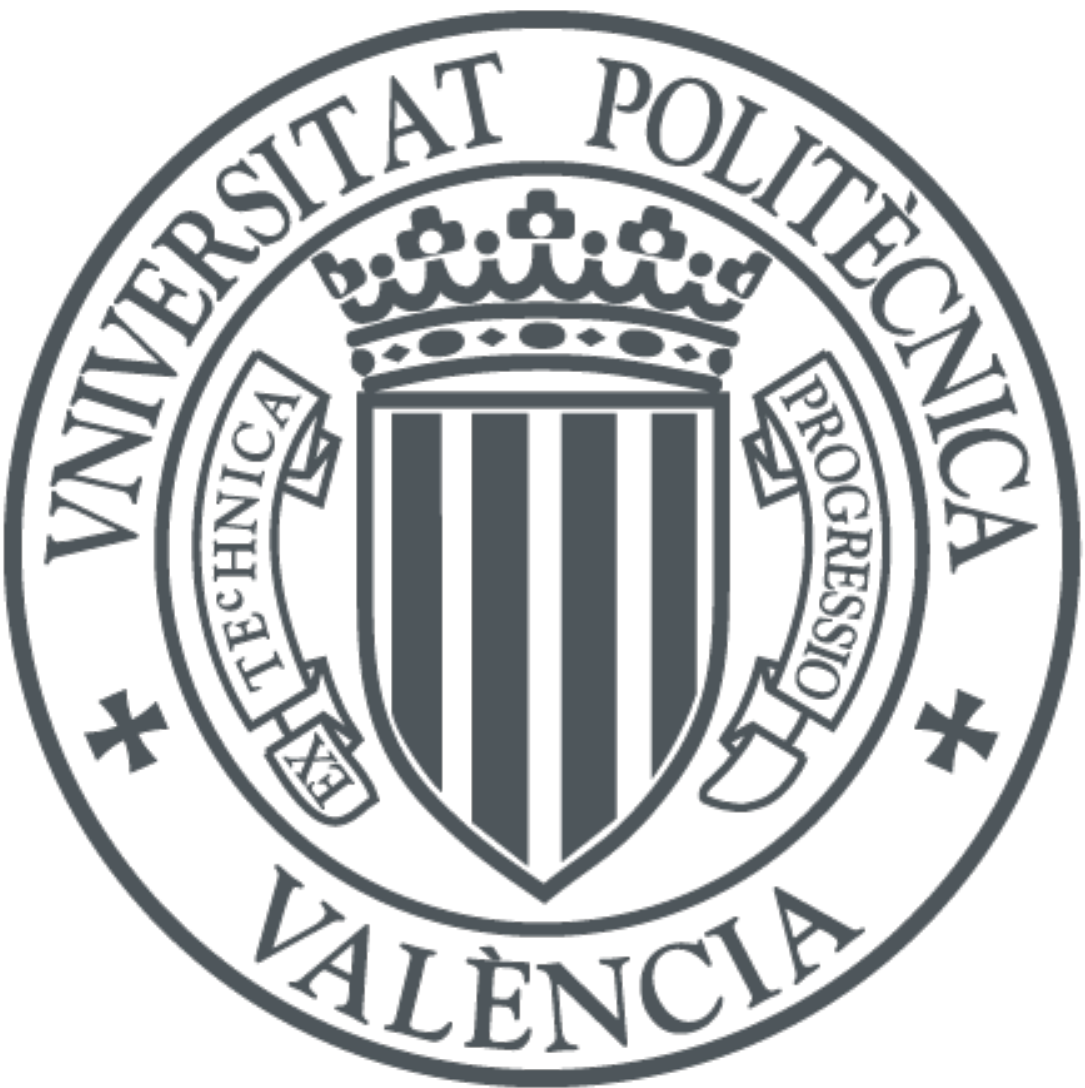

The final publication is available at

http://dx.doi.org/10.1039/c4cy01486c

Copyright Royal Society of Chemistry

Additional Information 


\title{
Palladium nanoparticles supported on graphene as catalyst for the dehydrogenative coupling of hydrosilanes and amines.
}

\author{
Juan Francisco Blandez, ${ }^{b}$ Iván Esteve-Adell, ${ }^{a}$ Mercedes Alvaro ${ }^{b}$ and Hermenegildo García*a \\ ${ }^{a}$ Instituto Universitario de Tecnología Química CSIC-UPV \\ $5^{b}$ Departamento de Química, Univ. Politécnica de Valencia, Av de los Naranjos s/n, 46022 Valencia, Spain. \\ E-mail: hgarcia@qim.upv.es
}

\begin{abstract}
10 Palladium nanoparticles (Pd NPs) have been supported on undopped and N-or B-doped graphenes (Gs) and these materials have been used as catalysts for the dehydrogenative coupling of hydrosilanes and amines to form silazanes. ${ }^{[1,2]}$ Working under optimal conditions, a conversion over $99 \%$ and selectivity of $84 \%$ were achieved for the reaction of dimethylphenylsilane with morpholine. In contrast, copper ( $\mathrm{Cu}$ NPs) or nickel nanoparticles (Ni NPs) supported on G did not promote the formation of the corresponding Si-N coupling product. It was found that $\mathrm{Pd} / \mathrm{G}$ performed better for this coupling than analogous catalysts in where Pd NPs were supported in active 15 carbon, multiwall carbon nanotubes or diamond NPs. Pd/G as catalyst has a wide substrate scope, including aliphatic and aromatic amines and mono or dihydrosilanes. Pd/G undergoes a gradual deactivation due to the growth and partial agglomeration of Pd NPs and aggregation of the $\mathrm{G}$ sheets, as observed by TEM.
\end{abstract}

\section{${ }_{20}$ Introduction.}

Due to the unique properties derived from its 2D morphology and the one-atom thickness, graphene $(\mathrm{G})$ offer unique properties as support for metal NPs. ${ }^{[3-7]}$ The interest of $\mathrm{G}$ and related material as supports in catalysis derives from its large specific surface area 25 that can be as large as $2500 \mathrm{~m}^{2} \times \mathrm{g}^{-1}[8]$ as well as from the strength of the interaction with the supported metal NPs arising from the overlap of the extended $\pi$ orbital of $G$ with the $d$ orbitals of transition metal atoms at the interface of the metal NP. G is constituted by one atom thick layer of $\mathrm{sp}^{2}$ carbons in hexagonal 30 arrangement and, therefore, the use of this type of material as supports represent the physical limit of miniaturization corresponding ideally to the minimum thickness for surface area $^{[9-11]}$ In addition, the properties of $G$ as support can be modified and the electronic effects tuned by the presence of 35 defects, oxygenated functional groups and heteroatoms. ${ }^{[12-17]}$ Theoretical calculations at the DFT level with G models with one carbon atom vacancy interacting with a transitions metal has shown that this type of defect characterized by dangling bonds on $\mathrm{G}$ is particularly suited to interact with metal atoms. ${ }^{[18]}$ Similar 40 type of computational calculations also indicate that heteroatoms on the $\mathrm{G}$ sheet can stabilize metal NPs. The so far reported data in the literature support that $\mathrm{G}$ is a unique material offering many opportunities to enhance the catalytic activity of metal NPs.

In this context, the present manuscript reports the catalytic 45 activity of doped G supported Pd NPs for the dehydrogenative coupling of hydrosilanes and amines (Eq. 1).

This reaction between hydrosilanes and amines has been reported in the literature with homogeneous catalysts using mainly Pd 50 complexes. ${ }^{[19,20]}$ Related to the dehydrogenative coupling of hydrosilanes and amines, the analogous reaction between hydrosilanes and alcohols has been recently reported to be performed using heterogeneous catalysts. ${ }^{[21]}$ In particular, it has been found that supported Au NPs can efficiently act as 55 heterogeneous catalysts for the reaction between hydrosilanes and alcohols, giving rise to the corresponding siloxane. Considering these precedents, it could also be that the coupling of hydrosilanes and amines can equally be promoted by supported metal NPs, Pd NPs being an obvious choice considering the ${ }_{60}$ known catalytic activity of Pd complexes for this reaction. ${ }^{[19,20]}$ The commercial interest of the resulting silazanes stems from their applications as silylating agents ${ }^{[22-25]}$ and for coating of material surfaces.

\section{Results and Discussion.}

65 A series of different Gs were prepared and used as supports of metals NPs. These materials were obtained, as reported, by pyrolysis under inert atmosphere of alginate or chitosan conveniently modified or not by boric acid. ${ }^{[26-28]}$ The resulting carbonaceous residues after pyrolysis can be exfoliated without 70 the need of additional chemical oxidation to form single and few layer $\mathrm{G}$ suspensions in different solvents. Scheme 1 illustrates the process followed for the synthesis of Gs.

Specifically the doping content and the distributions of the doped 75 element in two different sites were determined by quantitative XPS analysis. The layer morphology and the hexagonal arrangement of the $\mathrm{C}$ atoms were established by high resolution TEM imaging together with selected area electron diffraction. Figure 1 shows representative images of the samples to illustrate 80 the morphology of $\mathrm{G}$ and the particle size distribution of Pd NPs.

The single and few layers configuration of the suspended $G$ platelets was determined by AFM measurements of a statistically relevant number of $\mathrm{G}$ platelets. The $\mathrm{G}$ structure and the presence 85 of defects was established by Raman spectroscopy in where the characteristic graphitic (G) and defect (D) peaks appearing at about 1600 and $1350 \mathrm{~cm}^{-1}$ were observed. A quantitative 
estimation of the relative importance of defects for the various $G$ samples can be obtained from the relative intensity ratio of the $G$ versus the $\mathrm{D}$ bands. ${ }^{[28-32]}$ Overall, all the available characterization data of the $\mathrm{G}$ materials employed in the present 5 study are in agreement reported literature data that has previously established the formation of undoped or doped $G$ suspensions following the procedure employed in the present study.

In the initial stage of our work and in order to optimize the reactions conditions and select the most active catalyst, the 10 dehydrogenative coupling of dimethylphenylsilane and morpholine was chosen as model reaction. The influence of the reaction at temperatures from 60 to $120{ }^{\circ} \mathrm{C}$ was studied in the absence or in the presence co-solvents using different catalyst.

Table 1 summarizes the main results obtained in this screening 15 test. Gs in the absence of metals NPs (Table 1, entry 11) mainly catalyses disiloxane formation, the yield and the selectivity toward the desired product, 3a, being very low even after much longer times than those employed later. Also Cu NPs and Ni NPs at metal to substrate more ratio of $0.05 \mathrm{~mol} \%$ fail to promote the 20 dehydrogenative coupling. At high temperatures $(120 \mathrm{oC})$ and long reaction time $(24 \mathrm{~h})$, a notable hydrosilane 1a conversion was observed using $\mathrm{Cu} / \mathrm{G}$ as catalyst (Table 1, entry 9), but only trace amounts of the desired silazane $3 \mathrm{a}$ was detected under these conditions. This negative result contrasts with the catalytic 25 activity recently found for $\mathrm{Cu} / \mathrm{G}$ in the analogous dehydrogenative coupling of hydrosilanes with primary alcohols to render alcoxysilanes. Apparently the different properties of alcohols with regard amines in terms of nucleophilicity of the heteroatom and acidity of the hydrogens bonded to the 30 heteroatom should be responsible for this notable difference in the catalytic behavior of $\mathrm{Cu} / \mathrm{G}$ for these two related reaction.

In contrast, $\mathrm{Pd}$ containing catalysts promote the $\mathrm{Si}-\mathrm{N}$ coupling with high selectivity to product $3 \mathrm{a}$ at high substrate conversion. It was observed that, at the same Pd loading, the nature of the G and 35 the presence of dopant element plays a certain influence on the catalytic activity. It was found that $\mathrm{N}$-doping was detrimental for the catalytic activity, while the presence of B does not increase the activity with respect to undoped G. Accordingly Pd/G (Table 1 , entry 10) is apparently the best choice to promote this 40 coupling. As expected, the reaction temperature played a notable influence on the conversion and selectivity that increased as the temperature increased from 80 to $120 \mathrm{oC}$ (see Table 2, entries 1 , 2 and 3 ).

This influence of temperature on the initial reaction rate has 45 allowed us to estimate the activation energy of the process from the slope of the Arrhenius plot of $\log (\mathrm{k})$ versus 1/T (Figure 2).

The activation energy (Ea) estimated from the Arrhenius plot (Figure 2) was $22.9 \mathrm{~kJ} \times \mathrm{mol}^{-1}$ that is a low Ea value, but in the range of those determined for the $\mathrm{Cu}$-catalyzed dehydrogenative 50 coupling of hydrosilane and alcohols. Therefore, a suitable reaction temperature was $120{ }^{\circ} \mathrm{C}$. Working at this temperature and a $\mathrm{Pd} /$ substrate mol ratio of $0.05 \%$, full conversion 1a was obtained in $30 \mathrm{~min}$ (Table 1, entry 10).

With regard to the influence of co-solvents, it was observed that 55 the presence of 1,4-dioxane or toluene (Table 2, entries 4 and 5) is highly detrimental for the catalytic activity that disappear completely when any of these two solvents was present. Thus, reactions were carried out in the absence of co-solvents. The morpholine-hydrosilane mol ratio was also optimized. Initially, 60 an amine-hydrosilane mol ratio of 2 was selected, considering that the amine should play the role of reactant and solvent. But also, a 1-1 (Table 2, entry 7) and 1-2 mol ratios between morpholine and hydrosilane (Table 2, entry 8) were evaluated. It was found that a decrease in the mol ratio of morpholine leads to 65 a decrease in the yield of the coupling silazane product.

An additional experiment was carried out at $120{ }^{\circ} \mathrm{C}$ in the absence of co-solvent and $\mathbf{1 b - 2 a}$ mol ratio of 1-2, but using ten times lower amount of $\mathrm{Pd} / \mathrm{G}$ (Pd-1b mol ratio 0.003 mol\%). Full 1b conversion was also achieved in this experiment with very 70 high selectivity to $\mathbf{3 b}$, reaching a TON of product molecules per $\mathrm{Pd}$ atom of 33,300 although requiring much longer reaction time (24 h).

With the optimal conditions for the model reaction $\left(120{ }^{\circ} \mathrm{C}\right.$, no co-solvent, amine-silane mol ratio 2) the catalytic activity of

$75 \mathrm{Pd} / \mathrm{G}$ was compared to that of other Pd NPs supported in different carbon materials. The results are presented in Table 3.

Specifically in the present study we also checked the catalytic activity of Pd NPs supported on multiwall carbon nanotubes (MWCNTs) and reduced diamond nanoparticles (DH). MWCNTs 80 have many similarities with the structure of $\mathrm{G}$ and, as expected, the catalytic activity obtained using MWCNT as support was very similar (Table 3 , entry 5 ), the main difference should derive from the curvature of the $\mathrm{G}$ wall leading to the nanotube. In contrast, it has been found that $\mathrm{DH}$ offers a highly inert 85 environment to metal NPs, due to the lack of chemical reactivity and functional groups on the diamond surface. In this case 1a conversion was very similar to that achieved with $\mathrm{Pd} / \mathrm{G}$, but the selectivity to the desired product was very low (Table 3, entry 6). Besides allotropic carbon forms, active carbons have been 90 favorite supports in catalysis due to the large surface area and high adsorption capacity of these materials. The comparison of catalytic data shows that Pd NPs supported on active carbons (Table 3, entry 4) exhibit also a good performance, but with lower yield than that achieved with $\mathrm{Pd} / \mathrm{G}$. This comparative study 95 shows the superiority of $\mathrm{G}$ as support for this dehydrogenative coupling with respect to other carbon supports. In the literature there are precedents also showing the higher efficiently of supported metals NPs on G compared to other related carbons materials.(refs) This better performance has been attributed to the 100 combination of three factors, namely, large surface area of G, strong metal NPs-G interaction that may change the electronic density of the metal atoms at the metal NP-G interface and the presence of an extended $\pi$ orbital favoring adsorption of substrates and reagents near the metal NPs.(refs) It can be 105 assumed that the same effects could also operate in the present case.

Stability of $\mathrm{Pd} / \mathrm{G}$ as catalyst was studied by performing consecutive reuses of the same $\mathrm{Pd} / \mathrm{G}$ sample and determining the time-conversion plots for each run (Figure 3). After each 110 reaction, the catalyst was recovered by filtration, washed with hexane, dried at the ambient and submitted to another consecutive run. In addition, Pd content in the liquid phase after removal of the catalyst was also determined by ICP analysis to assess the possibility of $\mathrm{Pd}$ leaching from $\mathrm{Pd} / \mathrm{G}$ to the liquid phase.

115 As can be seen in Figure 3, a gradual decrease in activity was observed upon used, particularly between the second and the third 
use. Analysis of Pd in the liquid phase indicates that only the 0.12 $\%$ of the initial Pd has leached from $\mathrm{G}$ to the liquid phase and that four times reused $\mathrm{Pd} / \mathrm{G}$ still contains more than $99 \%$ of the $\mathrm{Pd}$ content of the fresh sample. This analytical data indicates that the 5 loss of Pd cannot be the cause of catalyst deactivation observed in Figure 3. This negligible percentage of Pd leaching is in favor of a strong $\mathrm{Pd} / \mathrm{G}$ interfacial interaction. We also performed a hot filtration test in which the reaction starts in the presence of catalyst, but then it is removed by filtration while the suspension 10 is still hot at a conversion of about $30 \%$ ( $5 \mathrm{~min})$. As it can be seen in Figure 4, the reaction stops when $\mathrm{Pd} / \mathrm{G}$ is removed from the reaction mixture. This indicates that there is no significant contribution of homogenous catalysis due to the small $\mathrm{Pd}$ leaching. These hot filtration data are in accordance with the low 15 amount of Pd leaching previously commented.

After four consecutive reuses, $\mathrm{Pd} / \mathrm{G}$ was characterized by TEM (Figure 5). Statistical analysis of the particle size distribution shows that after reuse of $\mathrm{Pd} / \mathrm{G}$ the dimension of $\mathrm{Pd} \mathrm{NPs}$ is between 3-7 nm. However, these images revealed also the 20 formation during the course of the reaction of some particles of much bigger size.7

The appearance of these large agglomerated Pd particles was observed in the almost deactivated catalyst, indicating that 25 particle size increase is, probably, one of the main causes of $\mathrm{Pd} / \mathrm{G}$ deactivation. Also in some areas the $2 \mathrm{D}$ morphology of $\mathrm{G}$ was not observed, and it seems that some $\mathrm{G}$ sheets become corrugated forming some 3D objects. This change in the morphology of $\mathrm{G}$ should also lead to catalyst deactivation due to the decrease in the 30 surface area of the $\mathrm{G}$ layers.

The scope of $\mathrm{Pd} / \mathrm{G}$ as catalyst for the coupling of hydrosilanes and amines was expanded by studying the reactivity of other aromatic and aliphatic mono and dihydrosilanes with primary and secondary aliphatic amines, as well as, aniline and acetamide.

35 The results obtained are shown in Table 4 and supporting information provides spectroscopic data of the resulting silazanes. As it can be see in Table 4, high conversions respect to the hydrosilane were obtained in all cases with selectivity values toward the expected silazane from good to high.

40 Due to the low boiling point, coupling with propylamine (2b) was carried out at $50{ }^{\circ} \mathrm{C}$, allowing the reaction to run for much lower time (Table 4, entries 2 and 5), achieving for 1a and triethylsilane (1b) high conversion and selectivity. Diphenylsilane (1c) also undergoes coupling with propylamine and the product for the

45 double coupling could be obtained with very high yields when the reaction was performed at $120{ }^{\circ} \mathrm{C}$. Aniline (2c) as substrate can also undergo dehydrogenative coupling with $\mathbf{1 a}$ and $\mathbf{1 b}$ to form the corresponding $\mathrm{N}$-phenylsilazanes $\mathbf{3 b}$ and $\mathbf{3 c}$. Even acetamide (4b) can react under the present conditions. In this case, bis-O,N50 disilane was the corresponding product observed in moderate yield at $120{ }^{\circ} \mathrm{C}$. Bis-O,N-disilane and related derivatives are among the preferred silylating reagents to perform the derivatisation of $\mathrm{OH}$ groups in sugars.

\section{Conclusions.}

${ }_{55}$ In the present manuscript it has been found that from the various doped and undoped G, Pd NPs supported on G is a suitable catalyst to promote the dehydrogenative coupling of hydrosilanes and primary or secondary amines with high conversion and good or high selectivity to the corresponding mono or disilazanes. The 60 reaction takes place even for aromatic amines and acetamide. In contrast, $\mathrm{Cu}$ NPs and Ni NPs do not promote this reaction. The use of $\mathrm{G}$ as support is advantageous with respect to other forms of carbons including active carbons, MWCNTs and DHNPs. Pd/G undergoes deactivation upon use, the most probable causes being ${ }_{65} \mathrm{Pd}$ NP agglomeration and aggregation of the G sheets.

\section{Acknowledgments.}

Financial support by the Spanish Ministry of Economy and Competitiveness (Severo Ochoa and CTQ-2012-32315) and ${ }_{70}$ Generaritat Valenciana (Prometeo 12/13) is grateful acknowledged. We also thank EU under the Being energy contract for partial funding. J. F. B. thanks the Universidad Politécnica de Valencia for a post graduate scholarship.

\section{${ }_{80}$ A Headings are the primary heading type}

The main text of the article should appear here. Headings and subheadings should be formatted using the relevant button from the "Apply Style" dialog box (see the RSC Tools toolbar above).

A tab stop has been set in the style to allow for easy indenting 85 of text (although the first paragraph and paragraphs that follow headings should not be indented).

\section{B Headings should always be subordinate to A headings}

1 You can also put lists into the text.

2 For this you should use the separately defined list style.

903 You can have bulleted or numbered lists of almost any kind.

4 However, you must remember to use a tab character to separate the symbol from the text.

\section{Inserting Graphics}

Use the buttons on the graphics dialog (accessed from the main 95 toolbar by pressing the "Insert Graphics" button) to insert the required template text. It is recommended that graphics are inserted once the text is completed, as the text after graphic insertion will cause the graphics to move unpredicatably. Graphics should be inserted at the top of the page where they are 100 first mentioned (unless they are equations, which appear in the flow of the text). Place the cursor at the beginning of the first line in either of the columns and press the required button.

\section{Conclusions}

The conclusions section should come at the end of article. 


\section{Notes and references}

${ }^{a}$ Address, Address, Town, Country. Fax: $X X X X X X X X X X$; Tel: $X X X X X X$ XXXX; E-mail:xxxx@aaa.bbb.ccc

${ }^{b}$ Address, Address, Town, Country. Fax: $X X X X X X X X X X$; Tel: $X X X X X X$ 5 XXXX; E-mail: xxxx@aaa.bbb.ccc

$\dagger$ Electronic Supplementary Information (ESI) available: [details of any supplementary information available should be included here]. See DOI: $10.1039 / \mathrm{b} 000000 \mathrm{x} /$

¥ Footnotes should appear here. These might include comments relevant 10 to but not central to the matter under discussion, limited experimental and spectral data, and crystallographic data.

1 A. Name, B. Name and C. Name, Journal Title, 2000, 35, 3523; A. Name, B. Name and C. Name, Journal Title, 2000, 35, 3523. 